\title{
COMPARAÇÃO DE ABORDAGENS ANALÍTICA, NUMÉRICA E EXPERIMENTAL PARA DETERMINAÇÃO DA VELOCIDADE DE FLUTTER EM UMA ASA IDEALIZADA
}

\author{
RODRIGUES, I.S. ${ }^{1}$, SANTIAGO, R.C. ${ }^{2}$ e ROCHA, A.D. ${ }^{3}$ \\ ${ }^{1}$ Graduando em Engenharia Aeroespacial na Universidade Federal do ABC \\ ${ }^{2}$ Professor Doutor na Universidade Federal do ABC \\ ${ }^{3}$ Professor Doutor na Universidade Federal do ABC \\ E-mail para contato: saveljevasig@ gmail.com
}

\begin{abstract}
RESUMO - Este trabalho compara modelos numéricos e analítico de determinação da velocidade de flutter com os dados obtidos em túnel de vento. A abordagem analítica se deu a partir do modelo de dois graus de liberdade, enquanto os modelos numéricos aplicados foram a solução aeroelástica proveniente do NX Nastran (DLM/FEM) e a interação fluido-estrutura de duas vias utilizando o software Ansys (CFD/FEM). Os resultados foram comparados com a velocidade de flutter observada experimentalmente, sendo que ambos os modelos numéricos foram capazes de prever a velocidade de flutter com precisão, enquanto o modelo clássico apresentou diferença na ordem de $36 \%$.
\end{abstract}

\section{INTRODUÇÃO}

Segundo Hodges e Pierce (2011), flutter é a uma instabilidade dinâmica associada com a interação de forças aerodinâmicas, elásticas e inerciais. Flutter também é autoinduzido e potencialmente destrutivo, na medida em que as forças aerodinâmicas se acoplam com os modos vibracionais aumentando a amplitude de movimento. Tanto Wright e Cooper (2015) como Hodges e Pierce (2011), tratam o problema de flutter na sua perspectiva linear, onde as superfícies sustentadoras produzem não só componentes estacionárias, como também pequenas perturbações não-estacionárias tratadas como harmônicas. Dessa forma, o fenômeno de flutter pode ser modelado matematicamente como uma discussão de estabilidade dinâmica. O modelo clássico de dois graus de liberdade apresentado na literatura possui diversas hipóteses simplificadoras. Dadas estas limitações, teorias como o doublet lattice method (DLM) foram desenvolvidas em conjunto com modelos de elementos finitos (FEM) para representar de forma mais fidedigna os fenômenos aeroelásticos. No entanto, de acordo com Wright e Cooper (2015), teorias como o DLM são falhas quando características aerodinâmicas não lineares estão presentes, como onda de choque, escoamentos com camada limite destruída, entre outros. Nesse contexto, as análises fluidodinâmicas computacionais (CFD) têm se popularizado devido o progresso tecnológico atual, sendo capazes de modelar efeitos viscosos, não-lineares e transônicos de forma acurada, inclusive acoplados, como o fenômeno de flutter. 


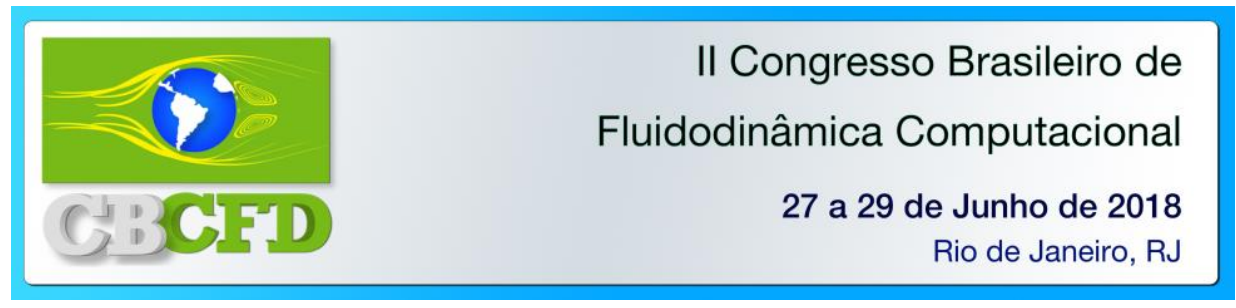

Tendo em vista as três metodologias de determinação da velocidade de flutter introduzidas acima (modelo de dois graus de liberdade e numéricos através do DLM/FEM e CFD/FEM), este trabalho teve como objetivo determinar a velocidade de flutter utilizando as três metodologias e comparar com resultados em túnel de vento. Como objetivo secundário, este trabalho servirá como material didático sobre aeroelasticidade para a equipe de aerodesign da Universidade Federal do ABC.

\section{METODOLOGIA}

O modelo de teste é uma placa de alumínio com dimensões de $230 \mathrm{~mm}$ x $115 \mathrm{~mm}$ e $0,4 \mathrm{~mm}$ de espessura, fixa em uma barra roscada M12 e posicionada com $2^{\circ}$ de ângulo de ataque, conforme ilustra a figura 1 .

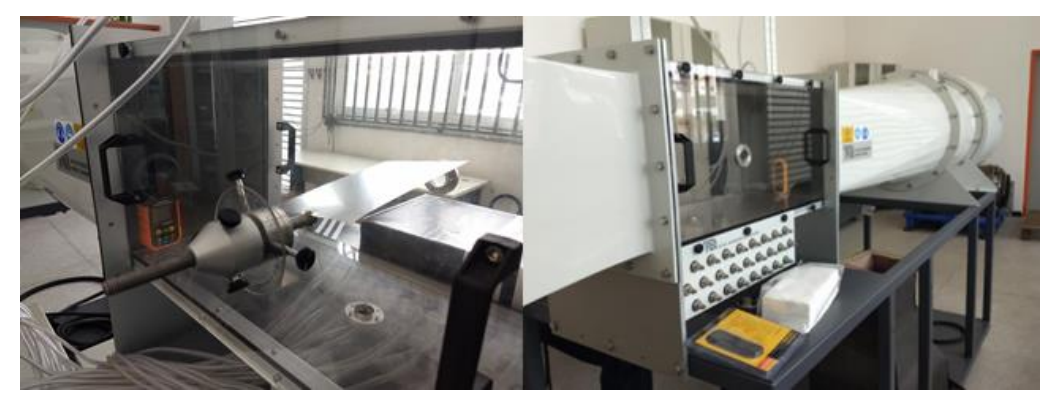

Figura 1 - Modelo de teste experimental

O teste experimental foi realizado no túnel de vento AF100 do fabricante TecQuipment na Universidade Federal do ABC. O túnel possui seção quadrada de $30 \mathrm{~cm}$ com $60 \mathrm{~cm}$ de comprimento, velocidade máxima de $32 \mathrm{~m} / \mathrm{s}$ e dois tubos de Pitot. As medições oriundas do tubo de Pitot foram aferidas com o anemômetro PM6252 da PeakMeter, sendo observadas flutuações na ordem de $1 \mathrm{~m} / \mathrm{s}$. Tendo como pré-requisito que a placa entrasse em flutter no túnel de vento, as dimensões foram propostas através de um estudo preliminar utilizando o modelo clássico de dois graus de liberdade, descrito na figura 2.

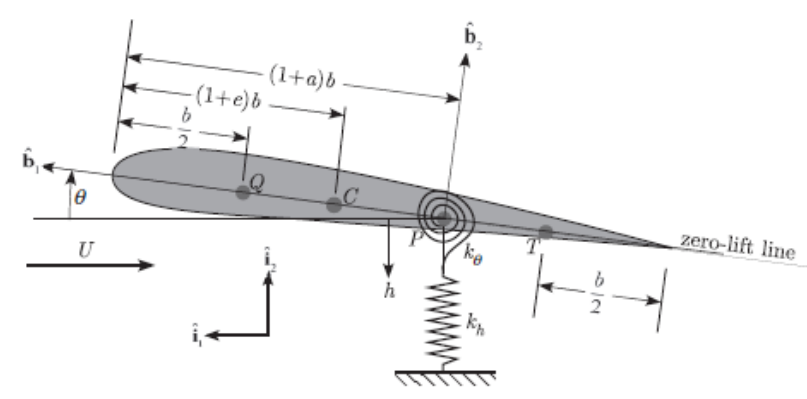

Figura 2 - Modelo clássico de dois graus de liberdade (Hodges e Pierce, 2011)

Neste modelo, segundo Wright e Cooper (2015), não se considera o acoplamento entre flexão e torção na matriz de rigidez, a distribuição de massa é uniforme e tanto o eixo elástico como o centro de massa estão posicionados no centro da corda. O método de solução aeroelástico aplicado foi o método $\mathrm{p}-\mathrm{k}$, sendo que o código foi validado através da comparação com o modelo apresentado por Wright e Cooper (2015). 


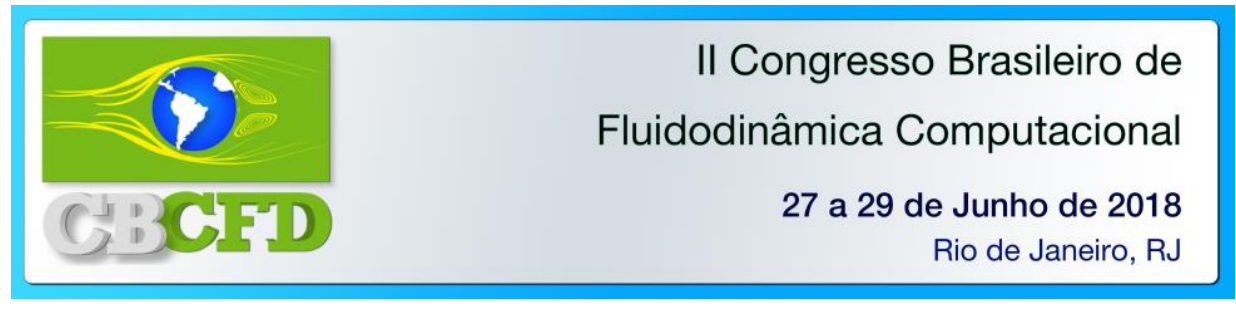

A Equação 1 representa a forma compacta já para a aplicação do método de autovalores e autovetores, onde A é a matriz de inércia, $\mathbf{B}$ e $\mathbf{D}$ de amortecimento aerodinâmico e estrutural e $\mathbf{C}$ e $\mathbf{E}$ de rigidez aerodinâmica e estrutural.

$$
\left\{\frac{\dot{q}}{\ddot{q}}\right\}-\left[\begin{array}{cc}
0 & \boldsymbol{I} \\
-\boldsymbol{A}^{-\mathbf{1}}\left(\rho V^{2} \boldsymbol{C}+\boldsymbol{E}\right) & -\boldsymbol{A}^{-\mathbf{1}}(\rho V \boldsymbol{B}+\boldsymbol{D})
\end{array}\right]\left\{\frac{q}{\dot{q}}\right\}=0 \text { ou } \dot{x}-\boldsymbol{Q} x=0
$$

Por sua vez, no modelo numérico desenvolvido no NX Nastran, foi utilizado para o modelo aerodinâmico à teoria DLM, para o modelo estrutural o elemento finito de placa QUAD4 e o método p-k para a solução do fenômeno de flutter. Foram simuladas duas malhas, sendo a segunda com o dobro de elementos da primeira, onde ambos os resultados foram iguais. A figura 3 ilustra o modelo elaborado no NX Nastran.

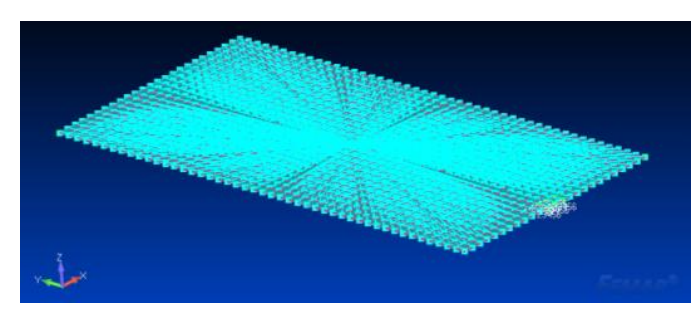

Figura 3 - Modelo aeroelástico no NX Nastran

No modelo de interação fluido-estrutura, primeiramente foi realizada uma análise 2D do perfil em três tamanhos de malha $\left(y^{+}=150,100 e 60\right)$, a fim de reproduzir a curva de sustentação e analisar a variação entre a malha menos e mais refinada, sendo esta variação aplicada nos resultados da malha tridimensional. Foi necessária esta abordagem dado o número limitado de volumes da versão disponibilizada. No modelo tridimensional foi utilizada a malha estruturada com $y^{+}$na ordem de 150 e crescimento na ordem de 1,2. Segundo o trabalho de Douvi et al. (2012), não foi detectada variação do coeficiente de sustentação com os modelos de turbulência para ângulos de ataque fora do estol. Nesse sentido, o modelo de turbulência utilizado foi o k-E realizable com função de parede EWT (Enhanced Wall Treatment, em inglês), método de solução Coupled, onde pressão e velocidade são calculadas conjuntamente, sendo o passo de tempo adotado de 0,01 s. O modelo estrutural foi desenvolvido com elemento sólido, onde se utilizou o elemento finito HEX20 com três elementos na espessura. O passo de tempo utilizado foi de $0,01 \mathrm{~s}$ e está de acordo com as frequências naturais da chapa. $\mathrm{O}$ acoplamento entre as análises foi forte, do inglês strong coupling. A velocidade foi aumentada até que foi observado um comportamento divergente da aceleração ao longo do tempo, a figura 4 ilustra as malhas tridimensionais.
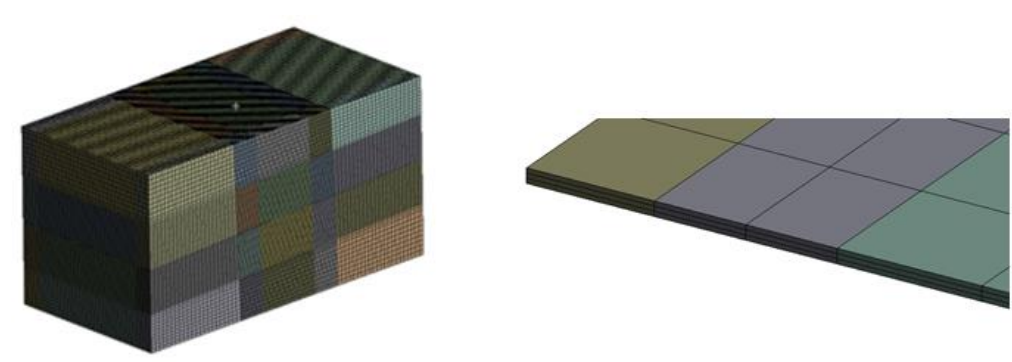

Figura 4 - Malha fluidodinâmica e estrutural no modelo de interação fluido estrutura 


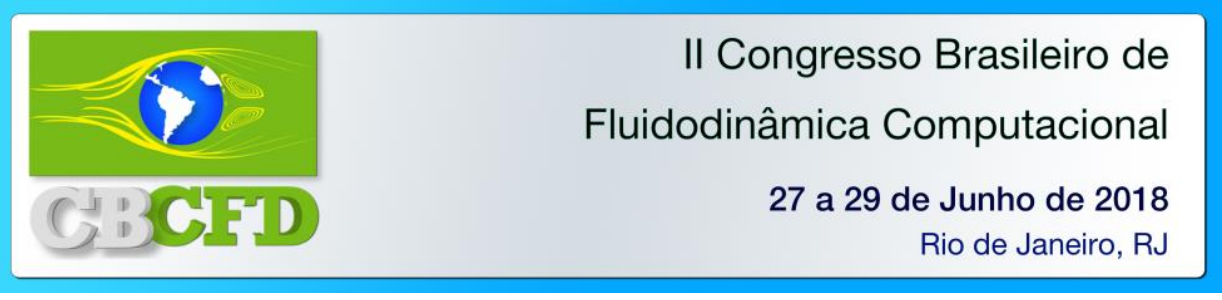

\section{RESULTADOS}

Os resultados obtidos por diferentes abordagens são apresentados na tabela 1 .

\begin{tabular}{c|c}
\multicolumn{2}{c}{$\begin{array}{c}\text { Tabela } 1 \\
\text { velocidades de } \text { flutter }\end{array}$} \\
\hline Método & Velocidade $(\mathrm{m} / \mathrm{s})$ \\
\hline Analítico & 10,20 \\
DLM/FEM & 15,96 \\
CFD/FEM & 17,00 \\
Experimental & 16,00 \\
\hline
\end{tabular}

Durante o teste no túnel de vento constatou-se que em $16,00 \mathrm{~m} / \mathrm{s}$ a placa entra em flutter de forma contínua, visualmente no modo torcional. Este resultado é corroborado com o resultado no NX Nastran, onde a velocidade flutter determinada está em 15,96 m/s. O modelo analítico apresentou velocidade de flutter em $10,20 \mathrm{~m} / \mathrm{s}$, sendo $36 \%$ menor que o valor de referência, no entanto o modelo foi capaz de prever o modo de flutter e o possível amortecimento crítico do modo em flexão. O resultado proveniente da interação fluidoestrutura apresentou velocidade de flutter em $17 \mathrm{~m} / \mathrm{s}$, no entanto a análise da variação da curva de sustentação mostrou que com a malha com $y^{+}$na ordem de 150 a sustentação é subdimensionada na ordem de $0,77 \mathrm{~m} / \mathrm{s}$.

\section{CONCLUSÃO}

Foi observado que as abordagens numéricas e analítica apresentam resultados coerentes com os resultados experimentais, sendo que os modelos numéricos foram precisos em relação ao cálculo. Mesmo apresentando diferença na ordem de 36\%, o modelo analítico não deve ser descartado, pois tem a sua aplicação em cálculos preliminares e comparativos.

Visto que o tempo de solução no NX Nastran foi de um minuto e no cálculo de interação no mínimo 40 horas, recomenda-se aplicar métodos de soluções similares ao DLM/FEM sempre que possível, pois será mais eficiente.

\section{REFERÊNCIAS}

DOUVI C. Eleni; TSAVALOS I. Athanasios; MARGARIS P. Dionissios. "Evaluation of the turbulence models for the simulation of the flow over a National Advisory Committee for Aeronautics (NACA) 0012 airfoil." Journal of Mechanical Engineering Research 4.3 (2012): 100-111.

HODGES, Dewey H.; PIERCE, G. Alvin. Introduction to structural dynamics and aeroelasticity. Cambridge, GBR: Cambridge University Press, 2011.

WRIGHT, Jan R.; COOPER, Jonathan E. Introduction to aircraft aeroelasticity. Reston, USA: American Institute of Aeronautics and Astronautics, c2015. 